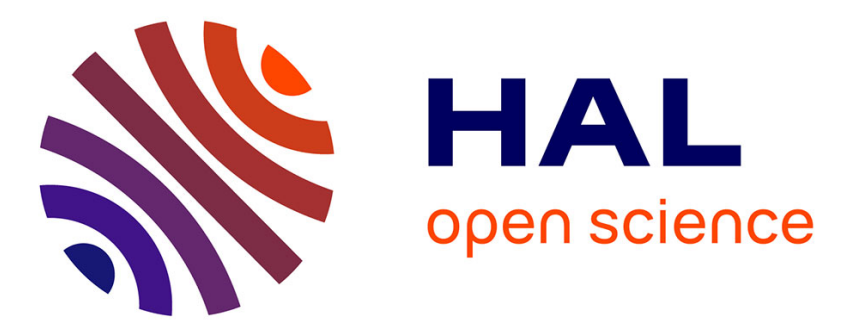

\title{
The reachable volume fraction in porous media in the vicinity of percolation threshold: a numerical approach used on multi-scale Boolean schemes
}

Johan Chaniot, Maxime Moreaud, Thierry Fournel, Jean-Marie Becker

\section{- To cite this version:}

Johan Chaniot, Maxime Moreaud, Thierry Fournel, Jean-Marie Becker. The reachable volume fraction in porous media in the vicinity of percolation threshold: a numerical approach used on multi-scale Boolean schemes. 2017 16th Workshop on Information Optics (WIO), Jul 2017, Interlaken, Switzerland. pp.1-3, 10.1109/WIO.2017.8038110 . hal-02095002

\section{HAL Id: hal-02095002 \\ https: / hal-ifp.archives-ouvertes.fr/hal-02095002}

Submitted on 10 Apr 2019

HAL is a multi-disciplinary open access archive for the deposit and dissemination of scientific research documents, whether they are published or not. The documents may come from teaching and research institutions in France or abroad, or from public or private research centers.
L'archive ouverte pluridisciplinaire HAL, est destinée au dépôt et à la diffusion de documents scientifiques de niveau recherche, publiés ou non, émanant des établissements d'enseignement et de recherche français ou étrangers, des laboratoires publics ou privés. 


\title{
The reachable volume fraction in porous media in the vicinity of percolation threshold: a numerical approach used on multi-scale Boolean schemes
}

\author{
Johan Chaniot $^{* \dagger}$, Maxime Moreaud*‡, Thierry Fournel ${ }^{\dagger}$, Jean-Marie Becker ${ }^{\dagger}$ \\ *IFP Energies nouvelles, Rond-point de l'échangeur de Solaize, BP 3, 69360 Solaize, France \\ †Université de Lyon, Université Jean Monnet de Saint Etienne, CNRS UMR 5516, \\ Laboratoire Hubert Curien, F-42000 Saint Etienne, France \\ ${ }^{\ddagger}$ MINES ParisTech, PSL-Research University, CMM, 35 rue Saint Honoré, 77305 Fontainebleau, France
}

\begin{abstract}
New morphological descriptors of complex porous networks are introduced and validated in this paper. These descriptors are based on the concept of "reachable volume fraction" here applied on multi-scale Boolean schemes; this fraction is computable for percolating spheres using step by step erosions of the porous network, providing information about the percolation strength of spherical particles with increasing radius. This process yields a critical radius, which, together with the dynamic reachable volume fraction provide a characterization of the porous media.
\end{abstract}

Keywords-Porous networks, Mathematical morphology, Percolation-threshold, Boolean schemes, Multi-scale, Geodesic distance transform, Reachable volume fraction

\section{INTRODUCTION}

Critical percolation-threshold is a crucial morphological descriptor for porous media in filtration systems and diphasic materials in conductive devices [1], [2]. Several approaches can be used to estimate this characteristic: probability formalism [3], [4], topology [5] or excluded volume [6], [2] for instance. It can be defined as a critical volume fraction [7] for an infinite volume size [8].

We propose a complementary approach of the critical percolation-threshold study, leading to new descriptors based on the reachable volume fraction as a function of the radius of a penetrating sphere. In this way, porous media may be characterized by such a discrete curve or its critical point (last one), that we will name descriptors in the sequel.

Firstly, we recall the link between these new descriptors and the critical percolation-threshold. Then we focus on the numerical study of single-scale Boolean schemes [9], [1], [10], for which we have a reference value, with a potential speed up of critical percolation-threshold computation. Finally we apply our new characterization to complex multi-scale Boolean schemes of platelets.

\section{Method}

\section{Critical radius}

For a Boolean scheme $A$, according to [11], the density $\theta$ of objects $A^{\prime}$ is given by:

$$
1-V_{v}=\exp \left(-\theta \cdot \bar{V}\left(A^{\prime}\right)\right)
$$

with $V_{v}$ the volume fraction of $\mathrm{A}$ and $\bar{V}\left(A^{\prime}\right)$ the average volume of objects $A^{\prime}$. For a Boolean scheme of spheres of radius $R, \bar{V}\left(A^{\prime}\right)=4 / 3 . \pi \cdot R^{3}$.
Let $A_{d}$ be a Boolean scheme defined as the dilation of $A$ by the ball $B(r)$,

$$
A_{d}=A \oplus B(r)
$$

This dilated Boolean scheme is equivalent to a Boolean scheme with a sphere of radius $R+r$, i.e. $\bar{V}\left(A_{d}^{\prime}\right)=4 / 3 \cdot \pi \cdot(R+r)^{3}$. Let $V_{v, d}$ be the volume fraction of the Boolean scheme $A_{d}$ defined as

$$
1-V_{v, d}=\exp \left(-\theta \cdot \bar{V}\left(A_{d}^{\prime}\right)\right)
$$

Using (1) and the expressions of $\bar{V}\left(A^{\prime}\right)$ and $\bar{V}\left(A_{d}^{\prime}\right)$ we can express $V_{v, d}$ as a function of $V_{v}$

$$
\begin{aligned}
1-V_{v, d} & =\exp \left(\ln \left(1-V_{v}\right) \cdot\left(\frac{R+r}{R}\right)^{3}\right) \\
& =\left(1-V_{v}\right)^{\alpha}
\end{aligned}
$$

where $\alpha=\left(\frac{R+r}{R}\right)^{3}$.

Henceforth a limit value of $r$ that we called the critical radius $r_{c}$ can be obtained when $V_{v, d}$ is equal to the critical percolation-threshold $\rho_{c}$.

$$
1-\rho_{c}=\exp \left(\ln \left(1-V_{v}\right) \cdot\left(\frac{R+r_{c}}{R}\right)^{3}\right)
$$

The critical radius $r_{c}$ is then defined by

$$
r_{c}=(\sqrt[3]{\beta}-1) \cdot R
$$

where $\beta=\frac{\ln \left(1-\rho_{c}\right)}{\ln \left(1-V_{v}\right)}$.

\section{Assessment of the critical percolation-threshold}

As in [1], [10], we consider that a realization percolates when there exists at least one connected component connecting two parallel faces of a representative cube. The critical percolation-threshold $\rho_{c}$ is numerically computed in [1] by dichotomic search. Several set of $2 N$ realizations of Boolean schemes are processed with different $V_{v} . \rho_{c}$ is obtained as the volume fraction of objects when exactly $N$ realizations percolate.

Our method avoids the dichotomic search and allows a timesaving trick. $\mathrm{N}$ realizations of Boolean scheme are generated and successive dilations applied on $A^{\prime}$ increase $V_{v}$. The 3D distance transform (see [12]) to which an increasing threshold is applied, allows these dilations. $\rho_{c}$ is then computed without 
generation of new realizations with different $V_{v}$.

We assess $\rho_{c}$ on 20 realizations of size $300^{3}$ of a single-scale Boolean scheme of spheres with $V_{v}=0.5$ and $R=10$. We obtain a critical percolation-threshold value equal to 0.914 for the complementary set in close relationship with the reference value 0.946 numerically found in [13]. This discrepancy finds its origin in the low precision computation due to the small ratio: volume size/ $(R+r)$ (this ratio is equal to 30 , whereas it is 400 in [13]).

\section{Numerical computation of the reachable volume fraction}

Our quantitative approach of the percolation allows the definition of new morphological descriptors. The computation process is described. $\mathrm{N}$ realizations of single-scale Boolean schemes of spheres with given $V_{v}$ and $R$ are generated. Let $I$ be one of these realizations. We focused in the percolation of the complementary set $I^{c}$. We erode $I^{c}$ by a spherical element with increasing radius $r$ and the reachable volume fraction $V_{r}$ is computed at each step. $V_{r}$ is defined as the summation of the volume fraction of all connected components which percolate,

$$
V_{r}=\sum_{i} V\left(C C_{i}\right) / V_{T}
$$

where $C C_{i}$ the $i^{t h}$ percolating connected component, $V\left(C C_{i}\right)$ its volume and $V_{T}$ the total volume. The term "reachable" represents then the accessibility for spherical particle which looks for percolating through the porous network. The successive erosions, efficiently computed by means of a geodesic distance map $D_{g}$ [12] (threshold of $D_{g}$ ), allow the simulation of a percolating sphere with increasing radius until $r_{c}$ is reached. In practical the algorithm's end is defined by $V_{r}$ equal to zero, the critical point is the coordinates which precede this "limit". The numerical $r_{c}$ is the abscissa of this critical point.

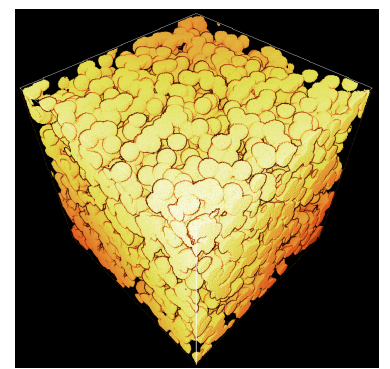

(a)

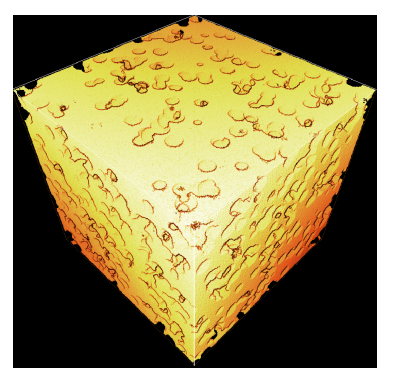

(b)
Fig. 1: (a) Realization of a Boolean scheme of spheres with $R=10$ and $V_{v}=0.5$ and (b) its complementary set.

\section{RESULTS AND DISCUSSION}

\section{Single-scale Boolean scheme}

First we assess the critical percolation-threshold $\rho_{c}$ by increasing the volume fraction $V_{v}$ on single-scale Boolean schemes of spheres. The reachable volume fraction $V_{r}$ can be computed until $r_{c}$ is reached in the vicinity of $\rho_{c}=0.946$. We applied our descriptors on Boolean schemes of spheres with $V_{v}=0.7$ and $R=10$. The volumes generated have a size of
$300^{3}$. For an aesthetic purpose Boolean schemes of spheres with $V_{v}=0.5$ and $R=10$ are shown. Figure 1 presents a realization of such a model and 2D slices of $I^{c}$ and distance map $D_{g}$ used to calculate erosion are shown figure 2 .

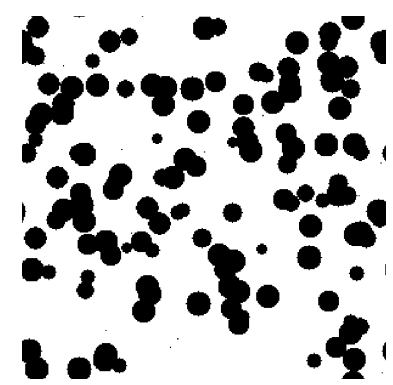

(a)

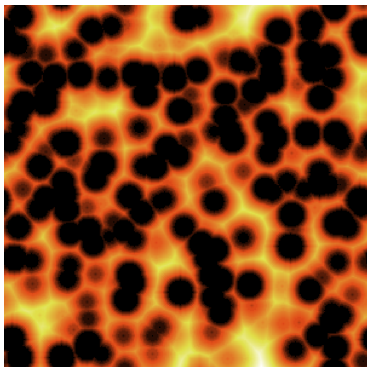

(b)
Fig. 2: (a) 2D slices of $I^{c}$ and (b) of the geodesic distance map $D_{g}$ for a realization with $R=10$ and $V_{v}=0.5$.

Computation of $V_{r}$ as a function of $r$ is presented in Table I. The third line presents the evolution of $V_{v}$ and shows its increase until the vicinity of $\rho_{c}$.

\begin{tabular}{|c|c|c|c|c|}
\hline $\mathrm{r}$ & 0 & 1 & 2 & 3 \\
\hline$V_{r}$ & 0.30 & 0.16 & 0.09 & 0.05 \\
\hline$V_{v}$ & 0.70 & 0.78 & 0.85 & 0.91 \\
\hline
\end{tabular}

TABLE I: Morphological descriptors of single-scale Boolean schemes of spheres with $V_{v}=0.7 R=10$.

Our morphological descriptors are then applied on singlescale Boolean schemes of sphero-cylinders. A sphero-cylinder is a cylinder with two hemispherical caps at each end, thus defined by two parameters $L$ the length of the cylinder and $R$ the radius for the hemispheres. Volume fraction $V_{v}$ and average volume $\bar{V}\left(A^{\prime}\right)$ are assigned the same as the Boolean scheme of spheres. Figure 3 presents a 3D image of one realization and the comparison of the descriptors with the previous scheme of spheres.

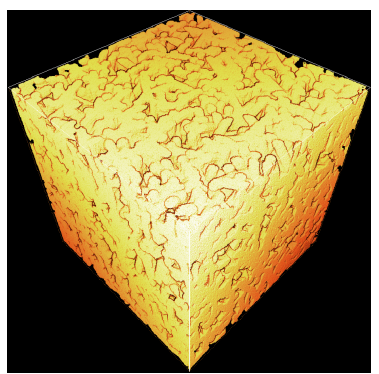

(a)

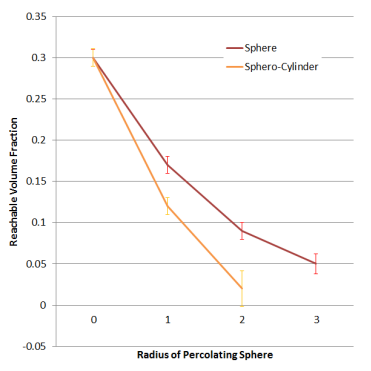

(b)
Fig. 3: (a) Single-scale Boolean scheme realization of spherocylinders with $R=5, L=47$ and $V_{v}=0.7$ and (b) the reachable volume fraction evolution for Boolean schemes of spheres $\left(V_{v}=0.7\right)$ (red) and sphero cylinders (yellow). 
The critical radius $r_{c}$ is equal to 2 and 3 for Boolean schemes of sphero-cylinders and spheres respectively. This difference results from the diameter of sphero-cylinders which is bigger than the one of spheres for a same volume. Here the diameter is defined as the maximal distance between two points of the object. Moreover the decrease of $V_{r}$ for sphero-cylinder's case is more important than for sphere. Both descriptors discriminate the schemes.

\section{Multi-scale Boolean scheme of aggregates of constant radius}

We applied our descriptors on complex multi-scale Boolean schemes of platelets' aggregates presented by Figure 4 which can be used to simulate digital microstructures of alumina catalysis supports [14].

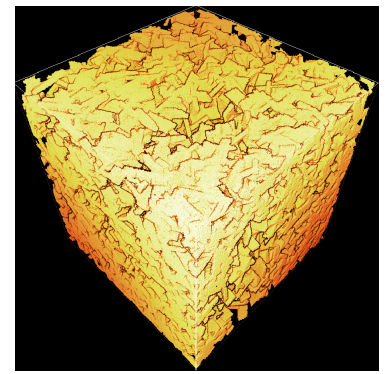

(a)

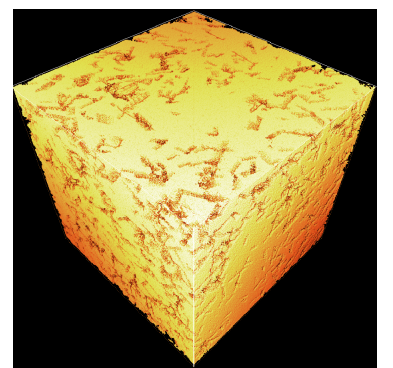

(b)
Fig. 4: (a) Multi-scale Boolean scheme of platelets with the radius of aggregate's spheres equals to 150 and (b) its complementary set.

This kind of Boolean scheme are defined by several parameters: shape of platelets, size of aggregates $\left(R_{\text {agg }}\right.$ radius of spheres), volume fraction of platelets inside $\left(V_{a g g}\right)$ and outside the aggregates. As seen previously the accessibility for an enlarging sphere is assessed and the reachable volume fraction $V_{r}$ is computed for two schemes differing by one parameter, in this case $R_{a g g}$. The first scheme presented Figure 4 is generated with $R_{a g g}=150$. The second one has a radius of aggregate's spheres equal to 50 . We compute the assessments on $N=20$ realizations for each scheme.

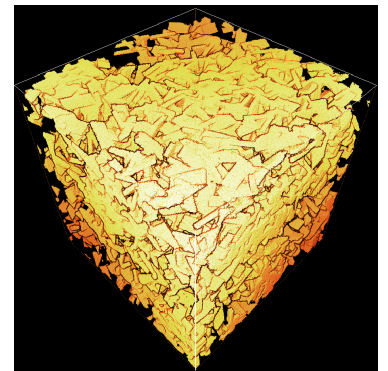

(a)

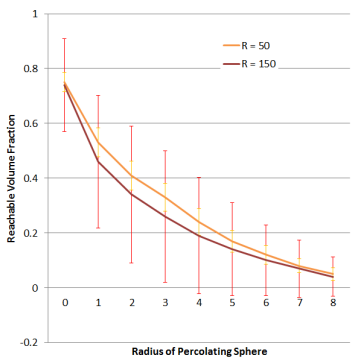

(b)
Fig. 5: (a) Multi-scale Boolean scheme of platelets with radius of aggregate's spheres equals to 50 and (b) the reachable volume fraction evolution for radius of aggregate's spheres equal to 50 (red) and 150 (yellow).
The critical radius $r_{c}$ is identical for both schemes and equal to 8 (abscissa of the critical point of both curves Figure 5). Nevertheless there is a noticeable difference in the evolution of $V_{r}$ on the graph Figure 5. Here the scalar descriptor does not be enough to distinguish the structures and the vectorial description is necessary.

\section{CONCLUSION}

We have presented an original approach to percolation which leads to new morphological descriptors. These descriptors allow the quantification of the volume accessibility of complementary set (here multi-scale Boolean schemes) according to specific percolating spheres with increasing radius. In a further work we will apply these new descriptors to the characterization of real porous lattices of catalysts.

\section{REFERENCES}

[1] D. Jeulin and M. Moreaud, "Percolation of multi-scale fiber aggregates," in $S 4 G$ (Stereology, Spatial Statistics and Stochastic Geometry) 6th International Conference, Prague, Republique Tchèque, 2006.

[2] M. Grujicic, G. Cao, and W. Roy, "A computational analysis of the percolation threshold and the electrical conductivity of carbon nanotubes filled polymeric materials," Journal of materials science, vol. 39, no. 14, pp. 4441-4449, 2004.

[3] M. E. Newman and R. M. Ziff, "Fast monte carlo algorithm for site or bond percolation," Physical Review E, vol. 64, no. 1, p. 016706, 2001.

[4] R. Parviainen, "Estimation of bond percolation thresholds on the archimedean lattices," J. Phys. A: Math. Theor, vol. 40, 2007.

[5] M. Moreaud, D. Jeulin, and S. Youssef, "Estimations of the critical percolation threshold from the zeros of connectivity number," International Congress of Stereology, Beijing, China. pp.400, 2011.

[6] I. Balberg, "Universal percolation-threshold limits in the continuum," Physical review B, vol. 31, no. 6, p. 4053, 1985.

[7] A. Delarue and D. Jeulin, "Multi-scale simulation of spherical aggregates," Image Analysis \& Stereology, vol. 20, no. 3, pp. 181-186, 2011.

[8] M. Rintoul and S. Torquato, "Precise determination of the critical threshold and exponents in a three-dimensional continuum percolation model," Journal of Physics A: Mathematical and General, vol. 30, no. 16, p. L585, 1997.

[9] D. Jeulin and M. Moreaud, "Multi-scale simulation of random spheres aggregates-application to nanocomposites," in 9th European Congress on Stereology and Image Analysis, Zakopane, Poland, vol. 1, 2005, pp. 341-348.

[10] Jeulin and Moreaud, "Percolation of random cylinder aggregates," Image Analysis \& Stereology, vol. 26, no. 3, pp. 121-127, 2011.

[11] G. Matheron, "Random sets and integral geometry," J. Wiley, New York, 1975.

[12] P. J. Toivanen, "New geodosic distance transforms for gray-scale images," Pattern Recognition Letters, vol. 17, no. 5, pp. 437-450, 1996.

[13] M. Moreaud, "Propriétés morphologiques multi-échelles et prévision du comportement diélectrique de nanocomposites," Ph.D. dissertation, École Nationale Supérieure des Mines de Paris, 2006.

[14] H. Wang, F. Willot, M. Moreaud, M. Rivallan, L. Sorbier, and D. Jeulin, "Numerical simulation of hindered diffusion in $\gamma$-alumina catalyst supports," Oil \& Gas Science and Technology-Revue d'IFP Energies nouvelles, vol. 72, no. 2, p. 8, 2017. 